\title{
BIENES SUJETOS A UN PROCEDIMIENTO SECUNDARIO DE INSOLVENCIA. COMENTARIO A LA SENTENCIA DEL TRIBUNAL DE JUSTICIA DE LA UNIÓN EUROPEA, DE 11 JUNIO 2015, NORTEL, C-649/13
}

\section{DEBTOR'S ASSETS FALLING WITHIN THE SCOPE OF THE EFFECTS OF THE SECONDARY PROCEEDINGS. JUDGMENT OF THE COURT (FIRST CHAMBER) OF 11 JUNE 2015, NORTEL, CASE C-649/13}

\author{
JULIANA RoDRÍGUEZ RODRIGO \\ Profesora Titular de Derecho Internacional Privado \\ Universidad Carlos III de Madrid \\ orcid ID: 0000-0002-5896-983X
}

Recibido: 02.07.2017 / Aceptado: 30.08.2017

DOI: https://doi.org/10.20318/cdt.2017.3898

\begin{abstract}
Resumen: En esta sentencia, el Tribunal de Justicia contesta a una cuestión prejudicial que plantea un órgano judicial francés en el marco de un procedimiento secundario de insolvencia. El tribunal remitente quiere saber qué órgano jurisdiccional es competente para determinar los bienes sujetos al procedimiento secundario -si el juez del procedimiento principal o el del secundario- y qué ley debe aplicar el tribunal competente para establecer cuáles son esos bienes -si la ley del procedimiento principal o la ley del procedimiento secundario-.
\end{abstract}

Palabras clave: insolvencia, procedimiento secundario de insolvencia, bienes del deudor.

Abstract: In that judgment, the Court answers a question referred by a French court in the context of secondary insolvency proceedings. The referring court wishes to know which court is competent to determine the assets subject to the secondary proceedings - if the judge in the main proceedings or the secondary court - and which law should be applied by the competent court to establish what those assets are - if the law of main procedure or the law of the secondary procedure.

Keywords: insolvency, secondary insolvency proceedings, debtor's assets.

Sumario: I. Hechos del caso. II. Regulación general en el Reglamento de insolvencia. 1. Competencia internacional. 2. Regulación de la insolvencia de un grupo de sociedad. 3. Materias objeto de regulación del Reglamento de insolvencia. 4. Procedimiento principal y procedimientos secundarios. III. Cuestiones planteadas. 1. Tribunal competente para determinar los bienes sujetos al procedimiento secundario de insolvencia. A. Competencia. B. Carácter de la competencia: exclusiva o alternativa. 2. Ley aplicable a la determinación de los bienes sujetos al procedimiento de insolvencia. IV. Conclusiones.

\section{Hechos del caso}

1. El presente asunto tiene su origen en un procedimiento de insolvencia planteado frente a un grupo de empresas denominado Nortel. Se trataba de un grupo dedicado a soluciones técnicas para redes 
de telecomunicaciones. Dentro del mismo, la empresa Nortel Networks Limited (NNL) era la propietaria de un gran número de filiales, entre ellas, NNSA, establecida en Francia.

Cuando el grupo quiebra, se abren procedimientos de insolvencia en Canadá, en los Estados Unidos y en la Unión Europea. El procedimiento principal en la Unión Europea se inicia en Reino Unido, para las sociedades del grupo establecidas en Europa.

Respecto de la empresa NNSA, se solicitó la apertura de procedimiento secundario en Francia.

2. Una vez se vendieron los activos del grupo, el administrador del procedimiento secundario entendió que la sociedad no podía contribuir al pago de los derechos sociales de los trabajadores de la empresa porque, pese a tener un saldo positivo derivado de la venta, los costes en los que había incurrido la compañía, por haber continuado con su actividad durante el procedimiento de insolvencia, habían sido mayores. Por este motivo, los trabajadores de NNSA reclamaron ante el tribunal francés su derecho a la parte proporcional de la venta de los activos del grupo que correspondía a NNSA y, consecuentemente, el pago de las cantidades adeudadas por la compañía. Los administradores mancomunados de la entidad, por su parte, impugnaron la competencia del tribunal francés al considerar que el único tribunal competente para dilucidar esta cuestión era la High Court of Justice de Inglaterra y Gales -órgano conocedor del procedimiento principal de insolvencia-.

Ante estas peticiones, el tribunal de comercio de Versailles (Francia) plantea la cuestión prejudicial que resuelve el Tribunal de Justicia en esta sentencia objeto de comentario. Lo que pregunta el tribunal remitente es si la competencia para determinar los bienes del deudor sujetos al procedimiento secundario, es exclusiva o alternativa a la competencia respecto del procedimiento principal. También pregunta el Derecho aplicable para determinar esos bienes sujetos, si debe ser el Derecho del procedimiento principal o el Derecho del procedimiento secundario.

\section{Regulación general en el Reglamento de Insolvencia}

3. El Reglamento 1346/2000 es la norma aplicable en este caso. Se trata del texto europeo relativo a procedimientos de insolvencia que se encontraba en vigor en esos momentos ${ }^{1}$. En la actualidad, este Reglamento ha quedado derogado por la entrada en vigor del vigente Reglamento 2015/848 ${ }^{2}$. Sin

\footnotetext{
1 Reglamento (CE) No 1346/2000 del Consejo, de 29 de mayo de 2000, sobre procedimientos de insolvencia, DO L 160, de 30 junio 2000, pp. 1-18.

Vid., entre otros, F. Garcimartín Alférez, "El Reglamento de insolvencia: una aproximación general", en AAVV, Cooperación jurídica internacional en materia civil. El Convenio de Bruselas, Consejo General del Poder Judicial, 2001, p. 231-352; J.L. VALLENS, "La maison mère d'un groupe, centre de intérêts principaux de ses filiales étrangères", Dalloz, 2006, pp. 793-798; J.L. Westbrook, “A global solution to multinational default", Michigan Law Review, vol. 98, n 7, 2000, pp. 2276-2328; D. BUREAU, "La fin d'un îlot de résistance. Le Règlement du Conseil relatif aux procédures d'insolvabilité" $R C D I P, \mathrm{n}^{\circ} 4$, octubredecembre 2002, p. 613-679; N. WATTE/V. MARQUETTE, "Le Règlement communautaire, du 29 mai 2000, relatif aux procedures d'insolvabilié", Rev.dr.com.belge, 2001, pp. 564-579; M. Virgós Soriano/F. Garcimartín Alférez, Comentario al Reglamento europeo de insolvencia", Thomson-Civitas, 2003; A.L. Calvo Caravaca/J. Carrascosa González, "Derecho concursal internacional", en A.L. Calvo Caravaca/J. Carrascosa González (dirs.), Derecho del comercio internacional, Colex, 2012, pp. 1835-2078; MOSS, G./FLETCHER, I./ISAACS, S. (eds.), The EC Regulation on insolvency proceeding. A commentary and annotated guide, [Oxford University Press], 2002; F. Garcimartín Alférez "Las normas de Derecho internacional privado de la ley concursal: algunas pautas para un correcto entendimiento", RJC, 2004, pp. 1269-1291; M. GuZMÁn PeCEs, "Globalización: Universalidad y fraccionamiento en los procedimientos de insolvencia. Un análisis desde el Derecho Internacional privado", Anuario Facultad de Derecho, III, Universidad de Alcalá, 2010, pp. 395-418; C. Esplugues Mota, "Procedimientos de insolvencia transfronterizos", Revista Seqüencia, no 52, julio 2006, pp. 9-34; M.J. Verdú CAÑETE, Procedimientos concursales comunitarios, Tirant Lo Blanch, Valencia, 2004; M. Llorente SÁncheZ-ArJonA, Tratamiento procesal de la insolvencia transfronteriza en la Unión Europea, Tirant Lo Blanch, Valencia, 2013; A. Espiniella MenÉndez, Procedimientos de insolvencia y grupos multinacionales de sociedades, Thomson, Navarra, 2006; G. AfFaKI (ed.), Cross-border insolvency and conflicto of jurisdictions, Bruylant, 2007; E. Beltrán SÁnchez, "El Reglamento de la Unión Europea sobre procedimientos de insolvencia", Tribunales de Justicia, abril 2001, pp. 31-43; J. RodRíGUEz RodRIGO, "El centro de intereses principales, como foro de competencia internacional en el Reglamento 1346/2000, en relación con empresas de un grupo de sociedades", Anuario de Derecho concursal, 2014, pp. 501536; G. García-Rostán Calvín, El proceso consurcal ante insolvencias conexas, Tirant Lo Blanch, 2015.

2 Reglamento (UE) 2015/848, del Parlamento Europeo y del Consejo, de 20 mayo 2015, sobre procedimientos de insolvencia, DOUE L 141, de 5 junio 2015, pp. 19-72. Este Reglamento ha empezado a ser aplicable, con carácter general, el 26 de junio de 2017 (art. 92).
} 
embargo, todo lo dicho en relación con el primero puede ser extrapolado al segundo, ya que, el contenido de ambas normas es similar en los preceptos estudiados en la sentencia. Por esta razón, hablaremos del Reglamento de insolvencia para aludir a la norma aplicada en el caso - Reglamento 1346/2000- y, al mismo tiempo, a la norma en vigor en la actualidad -Reglamento 2015/848-.

\section{Competencia internacional}

4. El Reglamento de insolvencia regula la competencia internacional, la ley aplicable y la validez extraterritorial de decisiones de procedimientos de insolvencia. Centrándonos en lo primero, la competencia internacional, el artículo 3 lleva ese título, y no es un error, esto es, no falta el término judicial para hablar de la competencia judicial internacional (Informe Virgós/Schmit, apartado 52) . $^{3}$ En efecto, existen países en los que los procedimientos de insolvencia son declarados por órganos que no son judiciales, por ello, el Reglamento habla de competencia internacional ${ }^{4}$. El propio artículo 2.d) del Reglamento 1346/2000 contemplaba esta posibilidad al definir tribunal como "el órgano judicial o cualquier otra autoridad competente de un Estado miembro habilitado para abrir un procedimiento de insolvencia o para adoptar decisiones en el curso del procedimiento"s - esta definición también está recogida en el artículo 2 número 6 del Reglamento 2015/848-.

5. El Reglamento de insolvencia regula la competencia internacional a través de foros que determinan el Estado cuyos órganos son los competentes. Una vez que estos foros resultan aplicables y conducen a un Estado, las normas procesales internas del mismo serán las aplicables para saber qué concreto órgano de ese Estado es el competente en el caso (Considerando 15 Reglamento 1346/2000 y Considerando 26 Reglamento 2015/848).

Pues bien, en el supuesto de que, en virtud del Reglamento, los tribunales españoles resultaran competentes, la Ley 22/2003, de 9 de julio, Concursal, será la que determine el concreto tribunal español competente ${ }^{6}$. En este sentido, el artículo 10.1 de esta Ley indica que los tribunales territorialmente competentes son los del lugar en el que la empresa tenga su centro de intereses principales. También estipula este precepto que si en España la empresa también tiene su domicilio social, pero lo tiene en un lugar distinto a aquel en el que se encuentra el centro de intereses principales, el acreedor solicitante podrá optar por demandar el concurso ante cualquiera de los Juzgados de lo Mercantil de esos dos lugares de España.

6. El foro del centro de intereses principales del deudor es el que prescribe el Reglamento en relación con un procedimiento principal de insolvencia. En este sentido, solo se aplicará si el centro de

\footnotetext{
${ }^{3}$ El Reglamento de insolvencia 1346/2000 es el sucesor del Convenio relativo a los procedimientos de insolvencia, hecho en Bruselas, el 23 de noviembre de 1995, Convenio que nunca entró en vigor. El Convenio de 1995 fue explicado a través de un Informe emitido por los profesores Virgós y Schmit y consensuado por todos los Estados contratantes. El legislador europeo quiso que el contenido del Reglamento de insolvencia fuera prácticamente idéntico al contenido de su antecesor Convenio de 1995 y, por ello, el Informe Virgós/Schmit se puede utilizar para explicar el contenido del Reglamento 1346/2000, del mismo modo que se habría utilizado en relación con el Convenio de 1995 (para conocer la sucesión de normas que precedieron al Reglamento de insolvencia vid., entre otros, E. Beltrán SÁnchez, "El Reglamento de la Unión Europea sobre procedimientos de insolvencia”, Tribunales de Justicia, abril 2001, pp. 32-34)

${ }^{4}$ En este sentido cabe citar, como ejemplo, el creditors voluntary winding-up del Reino Unido e Irlanda (F. GARCIMARTíN AlfÉrez, "El Reglamento de insolvencia: una aproximación general", en AAVV, Cooperación jurídica internacional en materia civil. El Convenio de Bruselas, Consejo General del Poder Judicial, 2001, p. 258).

5 Por su parte, la quiebra, el concurso de acreedores y otros procedimientos análogos que se encuentran excluidos expresamente del Reglamento 44/2001 son aquellos "basados, según las diversas legislaciones de las partes contratantes, en la situación de suspensión de pagos, la insolvencia o el quebranto del crédito del deudor, que implican una intervención de la autoridad judicial que conduce a una liquidación forzosa y colectiva de los bienes o, al menos, a un control por parte de dicha autoridad, es preciso, para que las resoluciones relativas a una quiebra se excluyan del ámbito de aplicación del Convenio, que sean consecuencia directa de la quiebra y se mantengan estrictamente dentro del marco de un procedimiento de liquidación de bienes o de suspensión de pagos con las referidas características" (STJCE de 22 de febrero 1979, Gourdain, 133/78, Rec. 1979, pg. 733, apartado 4). Esta definición introduce la necesaria intervención judicial en estos procedimientos, elemento éste que, como ya se ha comentado, no se exige en la definición de tribunal del artículo 2.d) del Reglamento 1346/2000 (D. BUREAU, "La fin d'un îlot de résistance. Le Règlement du Conseil relatif aux procédures d'insolvabilité” $R C D I P, \mathrm{n}^{\circ} 4$, octubre-decembre 2002, p. 625).

${ }^{6}$ BOE núm. 164, de 10 julio 2003
} 
intereses principales del deudor se encuentra en la Unión (Considerando 25 Reglamento 2015/848). No obstante, el artículo 3 del Reglamento de insolvencia, además de regular la competencia judicial internacional respecto de la apertura de un procedimiento principal, permite que se puedan abrir procedimientos secundarios en otros Estados miembros en los que el deudor tenga un establecimiento, eso sí, en estos casos, exclusivamente en relación a los bienes que el deudor posea en el territorio de esos países (art. 3.2).

\section{Regulación de la insolvencia de un grupo de sociedades}

7. El Reglamento 1346/2000 no regulaba nada en relación con los procedimientos de insolvencia de empresas que pertenecen a un grupo de sociedades, esto es, no indicaba nada sobre si se podría solicitar la insolvencia de un grupo entero de sociedades, o si habría que ir sociedad por sociedad, ni sobre las posibles repercusiones de la declaración de insolvencia de la matriz en relación con las filiales y viceversa?

El legislador europeo no previó nada al respecto debido a las dificultades jurídicas que una centralización competencial podría suponer al grupo de sociedades y, también, por la inseguridad que supondría un procedimiento único en relación con las diferentes sociedades regidas por distintos ordenamientos extranjeros y con dispares vínculos con sus propios acreedores ${ }^{8}$.

Esta ausencia de regulación ha llevado a muchos tribunales europeos a considerar que el centro de intereses principales de las filiales del grupo se encontraba donde la matriz tenía su administración central para, así, acumular la competencia ante el mismo tribunal competente en relación con la matriz9. De esta manera, en un mismo procedimiento se podría resolver la situación de insolvencia del grupo.

8. En la actualidad, el Reglamento $2015 / 848$ sí recoge una regulación específica para el grupo de sociedades en el Capítulo V. Desde el principio, se hace referencia al concepto "grupo de sociedades" para definirlo como "una empresa matriz y todas sus empresas filiales" (art. 2 número 13) y, después, en los artículos 56 y siguientes, el legislador europeo recoge las características del procedimiento de insolvencia en las distintas sociedades del grupo. En este Capítulo V, merece la pena destacar el deber de cooperación y de información que debe existir entre los administradores concursales de los distintos procedimientos abiertos y entre los órganos jurisdiccionales competentes en todos ellos (Considerando 52).

\section{Materias objeto de regulación del Reglamento de insolvencia}

9. El Reglamento no regula los límites de la jurisdicción en esta materia, es decir, no determina si es posible utilizar la vis atractiva concursus que existe en algunos ordenamientos y que permite conocer, junto con el estricto procedimiento de insolvencia, todas las acciones derivadas del mismo.

10. Como el Reglamento no dice nada, para saber qué cuestiones pueden ser sometidas a la jurisdicción del tribunal competente se propone utilizar la definición que el Tribunal de Justicia ha dado de "quiebra, convenios entre quebrado y acreedores y demás procedimientos análogos" como materia excluida del Convenio de Bruselas de 1968 (actual Reglamento 1215/2012) ${ }^{10}$.

\footnotetext{
7 A. Espiniella Menéndez, Procedimientos de insolvencia y grupos multinacionales de sociedades, Thomson, Navarra, 2006, p. 43

8 J.L. VAlLENS, "La maison mère d'un groupe, centre de intérêts principaux de ses filiales étrangères", Dalloz, 2006, p. 796. En este sentido de la inconveniencia de aplicar el universalismo a los grupos de sociedades, J.L. WestBrook, "A global solution to multinational default”, Michigan Law Review, vol. 98, nº 7, 2000, p. 2312.

9 J.L. VALLENS, "La maison mère d'un groupe, centre de intérêts principaux de ses filiales étrangères", Dalloz, 2006, p. 796.

10 N. Watte/V. Marquette, "Le Règlement communautaire, du 29 mai 2000, relatif aux procedures d'insolvabilié", Rev.dr. com.belge, 2001, p. 566

Convenio relativo a la competencia judicial y a la ejecución de resoluciones judiciales en materia civil y mercantil, hecho en Bruselas el 27 de septiembre de 1968, BOE núm. 24, de 28 enero 1991, p. 2910 (DO núm. C27, de 26 enero 1998).

Reglamento (UE) No 1215/2012 del Parlamento Europeo y del Consejo, de 12 de diciembre de 2012, relativo a la competencia judicial, el reconocimiento y la ejecución de resoluciones judiciales en materia civil y mercantil, DOUE L 351, de 20 diciembre 2012, pp. 1-32.
} 
Según el órgano jurisdiccional europeo, "Considerando que, por lo que se refiere a las quiebras, los convenios entre quebrado y acreedores y demás procedimientos análogos, que son procedimientos basados, según las diversas legislaciones de las partes contratantes, en la situación de suspensión de pagos, la insolvencia o el quebranto del crédito del deudor, que implican una intervención de la autoridad judicial que conduce a una liquidación forzosa y colectiva de los bienes o, al menos, a un control por parte de dicha autoridad, es preciso, para que las resoluciones relativas a una quiebra se excluyan del ámbito de aplicación del Convenio, que sean consecuencia directa de la quiebra y se mantengan estrictamente dentro del marco de un procedimiento de liquidación de bienes o de suspensión de pagos con las referidas caracteristicas" $" 11$.

En efecto, el carácter complementario que deben tener estas normas, el actual Reglamento 1215/2012 y el Reglamento 1346/2000, lleva a interpretar que las materias en cuestión excluidas del primero se encuentran incluidas en el segundo (Informe Virgós/Schmit, apartado 77). En este sentido también se pronuncia el Tribunal de Justicia en la sentencia objeto de comentario (apartado 26), pero, con una interpretación estricta que debe realizarse del ámbito de aplicación del Reglamento de insolvencia y, por ello, teniendo en cuenta que las acciones cubiertas por esta norma son "las que emanan directamente de un procedimiento de insolvencia y las que están estrechamente relacionadas con él (en lo sucesivo, acciones conexas)" (apartado 27).

\section{Procedimiento principal y procedimientos secundarios}

11. Los tribunales del lugar en el que el deudor tiene el centro de intereses principales serán competentes para conocer del procedimiento principal de insolvencia, procedimiento que se va a seguir sobre todos los bienes del deudor, independientemente de dónde se encuentren, en el mismo Estado miembro, en otro Estado miembro o en un tercer Estado. Así, el Considerando 12 Reglamento 1346/2000 y Considerando 23 del Reglamento 2015/848 disponen que "[...] Dichos procedimientos tienen alcance universal y su objetivo es abarcar todos los bienes del deudor [...]"12.

12. En ese procedimiento podrán participar todos los acreedores del deudor, de nuevo, independientemente de dónde tengan sus domicilios ${ }^{13}$. En efecto, el procedimiento de insolvencia regulado por el Reglamento es de carácter universal, tanto en relación con la masa activa (todos los bienes del deudor se encuentran sujetos a este procedimiento) como en relación con la masa pasiva (todos los acreedores pueden participar en él), como respecto a la ley aplicable (una única ley, la lex fori concursus) ${ }^{14}$.

11 STJCE 22 febrero 1979, Gourdain, 133/78, Rec. 1979, p. 733, apartado 4. M. Virgós Soriano/F. Garcimartín AlfÉrez, Comentario al Reglamento europeo de insolvencia”, Thomson-Civitas, 2003, p. 62

12 En este sentido se pronuncian el Informe Virgós/Schmit, apartado 73; M. Virgós Soriano/F. Garcimartín Alférez, Comentario al Reglamento europeo de insolvencia", Thomson-Civitas, 2003, p. 25; A.L. CAlvo CARAVACA/J. CARRASCOSA GonZÁlez, "Derecho concursal internacional", en A.L. Calvo Caravaca/J. Carrascosa GonzÁlez (dirs.), Derecho del comercio internacional, Colex, 2012, p. 1935; I. Fletcher, "Scope and Jurisdictioin”, en G. Moss/I. Fletcher/S. IsAaCs (eds.), The EC Regulation on insolvency proceedings. A commentary and annotated guide, Oxford University Press, 2002, p. 41; C. Esplugues Mota, "Procedimientos de insolvencia transfronterizos", Revista Seqüência, no 52, julio 2006, p. 12; M.J. Verdú CAÑETE, Procedimientos concursales comunitarios, Tirant Lo Blanch, Valencia, 2004, p. 53. Sin embargo, otros autores consideran excesiva la extensión del procedimiento a los bienes situados fuera de la Unión Europea (N. Watte/V. Marquette, "Le Règlement communautaire, du 29 mai 2000, relatif aux procedures d'insolvabilié", Rev.dr.com.belge, 2001, p. 572). Lo anterior no obstaría a que el órgano competente de un Estado miembro pidiera la colaboración del deudor en relación con bienes situados en terceros países (N. WATtE/V. MARQUETTE, "Le Règlement communautaire, du 29 mai 2000, relatif aux procedures d'insolvabilié", Rev.dr.com.belge, 2001, p. 573).

13 Informe Virgós/Schmit, apartado 73; I. Fletcher, "Scope and Jurisdictioin", en G. Moss/I. Fletcher/S. IsAacs (eds.), The EC Regulation on insolvency proceedings. A commentary and annotated guide, Oxford University Press, 2002 , p. 41.

14 M. Virgós Soriano/F. Garcimartín Alférez, Comentario al Reglamento europeo de insolvencia", Thomson-Civitas, 2003, p. 25; F. Garcimartín AlfÉrez, "El Reglamento de insolvencia: una aproximación general", en AAVV, Cooperación jurídica internacional en materia civil. El Convenio de Bruselas, Consejo General del Poder Judicial, 2001, p. 246; M. LLORENTE SÁnchez-Arjona, Tratamiento procesal de la insolvencia transfronteriza en la Unión Europea, Tirant Lo Blanch, Valencia, 2013, p. 102 (este último, en relación con la aplicación de la lex fori al procedimiento).

El artículo 32 del Reglamento 1346 y el artículo 45 del Reglamento 848 indican la universalidad en relación con la masa pasiva. 
13. Ahora bien, el Reglamento responde a un universalismo mitigado en cuanto a la ley aplicable al mismo, ya que recoge determinadas reglas particulares que atenúan este carácter universal del procedimiento ${ }^{15}$. Así, por un lado, se quiebra la universalidad en la ley aplicable por cuanto existen cuestiones particulares a las que no se va a aplicar la ley del foro ${ }^{16}$. Por otro lado, también se quiebra la universalidad en el procedimiento, en la medida en que, junto al procedimiento principal, pueden abrirse procedimientos secundarios en los Estados donde el deudor tenga un establecimiento (art. 3.2) ${ }^{17}$. Además, en el caso de que no se haya iniciado un procedimiento principal, se podría solicitar la apertura de un procedimiento autónomo bajo determinados presupuestos (art. 3.4).

14. En relación con esto último, el Capítulo III del Reglamento regula los procedimientos secundarios de insolvencia, procedimientos que pueden abrirse, junto con el principal, en los Estados miembros donde el deudor tenga un establecimiento y en relación con los bienes que existan en esos países -los acreedores que pueden concurrir a esos procedimientos, sin embargo, no tienen que ser locales, pueden ser europeos o de terceros Estados (art. 32 Reglamento 1346 y art. 45 Reglamento 848)- ${ }^{18}$.

Los procedimientos secundarios son procedimientos territoriales, los cuales, a su vez, pueden ser, también, autónomos o independientes cuando se abren sin la existencia de un procedimiento principal ${ }^{19}$. Por lo tanto, los procedimientos territoriales pueden ser secundarios, cuando existe un procedimiento principal, o independientes, cuando no existe un procedimiento principal ${ }^{20}$. El Considerando

15 M. Virgós Soriano/F. Garcimartín Alférez, Comentario al Reglamento europeo de insolvencia”, Thomson-Civitas, 2003, p. 26.

${ }_{16}$ M. Virgós Soriano/F. Garcimartín Alférez, Comentario al Reglamento europeo de insolvencia”, Thomson-Civitas, 2003, pp. 91-141. En efecto, la ley aplicable al procedimiento de insolvencia será la ley del foro, el Derecho nacional concursal del foro (art. 4 Reglamento 1346 y art. 7 Reglamento 848), sin embargo, en relación con determinadas situaciones particulares, dicha ley nacional puede no ser la aplicada. Esto es lo que ocurre, por ejemplo, cuando el Reglamento dispone que los efectos del procedimiento de insolvencia en relación con un contrato sobre un bien inmueble quedarán regulados por la ley del país en el que el bien se encuentre situado (art. 8 Reglamento 1346 y art. 11 Reglamento 848). En este punto, la universalidad se rompe, no todos los bienes del deudor van a quedar regulados por la misma ley (M. Llorente SÁnchEz-ArjonA, Tratamiento procesal de la insolvencia transfronteriza en la Unión Europea, Tirant Lo Blanch, Valencia, 2013, p. 117; C. EsPLUGUES MotA, "Procedimientos de insolvencia transfronterizos", Revista Seqüência, n 52, julio 2006, p. 18).

17 "Establecimiento" es, a efectos del Reglamento 1346/2000, "todo lugar de operaciones en el que el deudor ejerza de forma no transitoria una actividad económica con medios humanos y bienes" (art. 2.g) y, en el marco del Reglamento 2015/848, "todo lugar de operaciones en el que un deudor ejerza o haya ejercido, en los tres meses anteriores a la apertura del procedimiento principal de insolvencia, de forma no transitoria una actividad económica con medios humanos y materiales" (art. 2 número 10)

18 M. Virgós Soriano/F. Garcimartín Alférez, Comentario al Reglamento europeo de insolvencia", Thomson-Civitas, 2003, p. 27 y p. 175; N. Watte/V. Marquette, "Le Règlement communautaire, du 29 mai 2000, relatif aux procedures d'insolvabilié”, Rev.dr.com.belge, 2001, p. 566; M. Llorente SÁnCHEZ-ArJona, Tratamiento procesal de la insolvencia transfronteriza en la Unión Europea, Tirant Lo Blanch, Valencia, 2013, p. 77; A. Espiniella MenÉndez, Procedimientos de insolvencia y grupos multinacionales de sociedades, Thomson, Navarra, 2006, p. 150.

Estos procedimientos secundarios, en el marco del Reglamento 1346, debían ser procedimientos de liquidación (art. 3.3) -a diferencia de los principales que pueden ser de liquidación o de saneamiento de la empresa- (F. Garcimartín Alférez, "El Reglamento de insolvencia: una aproximación general", en AAVV, Cooperación jurídica internacional en materia civil. El Convenio de Bruselas, Consejo General del Poder Judicial, 2001, p. 247; F. Garcimartín Alférez "Las normas de Derecho internacional privado de la ley concursal: algunas pautas para un correcto entendimiento", RJC, 2004, p. 1276)

19 M. Virgós Soriano/F. Garcimartín Alférez, Comentario al Reglamento europeo de insolvencia”, Thomson-Civitas, 2003, pp. 27-28.

20 Informe Virgós/Schmit, apartado 25; M. LloRente SÁnCHEZ-ArJonA, Tratamiento procesal de la insolvencia transfronteriza en la Unión Europea, Tirant Lo Blanch, Valencia, 2013, pp. 76-77.

Los procedimientos independientes se pueden iniciar en dos circunstancias que difieren según hablemos del Reglamento 1346 o del Reglamento 848. Así, en virtud del primero, esas circunstancias recogidas en el artículo 3.4 son: 1) no se puede abrir un procedimiento principal según la ley del Estado en el que el deudor tiene su centro de intereses principales ( $a d$ ex., porque esa ley no permite la apertura de un procedimiento de insolvencia contra un deudor no comerciante (I. FLETCHER, "Scope and Jurisdictioin", en G. Moss/I. Fletcher/S. IsAaCs (eds.), The EC Regulation on insolvency proceedings. A commentary and annotated guide, Oxford University Press, 2002, p. 43; N. Watte/V. Marquette, "Le Règlement communautaire, du 29 mai 2000, relatif aux procedures d'insolvabilié", Rev.dr.com.belge, 2001, p. 574); 2) el procedimiento territorial ha sido iniciado por un acreedor con domicilio, sede o residencia habitual en ese Estado donde el deudor tiene un establecimiento o cuando tiene un crédito derivado de la explotación de dicho establecimiento. De este modo, la apertura del procedimiento independiente se limita, salvo en el caso de que no pudiera iniciarse un procedimiento principal, a los acreedores locales y a los acreedores del establecimiento local del deudor (Considerando 17); sin embargo, una vez iniciado el procedimiento, podrán participar en el mismo todos los acreedores interesados, independientemente de dónde se encuentren establecidos (Informe Virgós/Schmit, 
17 del Reglamento 1346 y el artículo 3.4 del Reglamento 848 estipulan también que, si después de la apertura del procedimiento autónomo se inicia un procedimiento principal, el primero se convierte en procedimiento secundario ${ }^{21}$.

La apertura de un procedimiento secundario se justifica por varias razones (Considerando 19 Reglamento 1346 y Considerando 40 Reglamento 848). Por un lado, para proteger intereses locales. En segundo lugar, porque puede haber casos en los que los bienes del deudor sean demasiado complejos para poder administrarse de forma unitaria. Por último, porque las diferencias entre los Derechos nacionales en materia concursal pueden ser tan grandes que pueden existir dificultades a la hora de que se apliquen dichas legislaciones en otros Estados miembros donde el deudor tenga bienes.

\section{Cuestiones planteadas}

\section{Tribunal competente para determinar los bienes sujetos al procedimiento secundario de insol- vencia}

\section{A. Competencia}

15. El Tribunal de Justicia, alegando reiterada jurisprudencia en relación con la competencia del artículo 3.1 del Reglamento1346/2000, considera que el efecto útil del mencionado Reglamento de insolvencia lleva a aplicar por analogía lo dicho en relación con el artículo 3.1 respecto de la competencia en un procedimiento secundario del artículo 3.2 de la norma -lo mismo cabe decir en relación con el Reglamento 2015/848-.

16. Por lo tanto, el tribunal competente en el procedimiento secundario, esto es, el tribunal del Estado donde el deudor tenga establecimiento, también será competente para resolver cuestiones anexas que pudieran plantearse relacionadas con él, como, por ejemplo, si tiene competencia para determinar los bienes del deudor afectos al procedimiento.

El Considerando 35 del Reglamento 848 así lo estipula al afirmar que el tribunal competente en el procedimiento de insolvencia lo será para conocer de las acciones que se deriven directamente del procedimiento y de todas las demás que guarden estrecha vinculación con éste.

\section{B. Carácter de la competencia: exclusiva o alternativa}

17. Una vez establecido que la competencia para determinar los bienes sujetos al procedimiento secundario la tiene el órgano competente en ese procedimiento, el Tribunal de Justicia estudia si esa competencia debe ser exclusiva o debe compartirse con el tribunal competente en el procedimiento principal.

Pues bien, la respuesta del Tribunal europeo es la segunda, esto es, esa competencia es concurrente o alternativa respecto del tribunal que está conociendo del procedimiento principal.

18. La razón fundamental que aporta el Tribunal de Luxemburgo es el hecho de que la decisión respecto de esos bienes del deudor que deben considerarse sujetos al procedimiento secundario, afecta al procedimiento principal puesto que se trataría de bienes que ya no podrían ser tenidos en cuenta en éste.

apartado 27; M. LloRente SÁnchez-ARJona, Tratamiento procesal de la insolvencia transfronteriza en la Unión Europea, Tirant Lo Blanch, Valencia, 2013, p. 78). En cambio, en el marco del actual Reglamento 848, mientras la primera circunstancia es similar a la que acabamos de mencionar del texto derogado, la segunda es diferente. En efecto, en segundo lugar, puede abrirse un procedimiento independiente del principal cuando lo inicia: 1) un acreedor cuyo crédito tenga su origen en la explotación de un establecimiento del deudor situado en el Estado donde se inicia el procedimiento secundario o que se esté relacionado con esta explotación; 2) una autoridad pública que tenga competencia, según la ley del Estado donde se encuentra el establecimiento, para la apertura del procedimiento.

21 Informe Virgós/Schmit, apartado 25. 
19. Si bien esta argumentación tiene todo el sentido, también es verdad que el atribuir competencia alternativa en este tema -como en cualquiera- puede tener como consecuencia negativa que se dicten resoluciones contradictorias al respecto. En este sentido, el órgano jurisdiccional europeo alega el artículo 25.1 del Reglamento 1346, en virtud del cual, los jueces están obligados a reconocer resoluciones anteriores dictadas por otros tribunales competentes en relación con estas cuestiones anexas, esto es, acciones que deriven directamente del procedimiento de insolvencia y guarden inmediata relación con éste -en el mismo sentido se pronuncia el artículo 32.1 del Reglamento 848-.

20. La universalidad del procedimiento conlleva que la resolución de apertura del mismo sea reconocida y ejecutada en todos los Estados miembros, de forma automática (art. 16 Reglamento 1346 y art. 19 Reglamento 848) y, por tanto, produzca en todos ellos los mismos efectos que le atribuye la ley del Estado del foro ${ }^{22}$.

En relación con el resto de resoluciones dictadas por el órgano competente -resoluciones relativas a cuestiones anexas que aparecen específicamente previstas en el segundo párrafo del artículo 25.1 del Reglamento 1346 y del artículo 32.1 del Reglamento 848, por ejemplo-, el Reglamento sigue estableciendo el reconocimiento automático pero, respecto de la ejecución, el texto europeo remite al régimen previsto con carácter general en materia civil y mercantil, esto es, al Convenio de Bruselas de 1968, en el caso del Reglamento 1346, y al Reglamento 1215/2012, en el caso del Reglamento 848. Por lo tanto, en la actualidad, en estos casos, se deben aplicar las reglas de procedimiento del Reglamento $1215 / 2012$ y las reglas de control de la decisión del Reglamento de insolvencia ${ }^{23}$.

El único motivo que aparece expresamente recogido en el Reglamento de insolvencia para no reconocer o ejecutar una resolución en esta materia es que el reconocimiento o la ejecución atente contra el orden público internacional del Estado requerido, en especial, cuando atente contra los principios fundamentales o contra los derechos y libertades individuales recogidos en la Constitución (art. 26 Reglamento 1346 y art. 32 Reglamento 848) ${ }^{24}$. En el marco del Reglamento 1346, además del orden público,

22 J.L. VALLENS, "L'exequatur des jugements étrangers de faillite après le Règlement communautaire sur les procedures d'insolvabilité", Petites affiches, $\mathrm{n}^{\circ}$ 18, 13 juin 2002, p. 16.

Algunos autores consideran que esa decisión de apertura del procedimiento de insolvencia debe ser pronunciada por un órgano judicial, todo ello, por la aplicación analógica en este punto del Convenio de Bruselas de 1968 (actual Reglamento 1215/2012) (J.L. Vallens, Petites affiches, $\mathrm{n}^{\mathrm{o}}$ 18, 13 juin 2002, p. 19). Lo anterior contradice la afirmación que se ha hecho más arriba de que el órgano que puede conocer de un procedimiento de insolvencia no tiene que ser necesariamente judicial.

La "resolución de apertura del procedimiento" no sólo es aquella que así se considera por el Derecho del foro, también debe ser entendida como tal, toda resolución dictada en el procedimiento de insolvencia del deudor, cuando implique el desapoderamiento del mismo y el nombramiento de un síndico (STJUE de 2 mayo 2006, Eurofood, C-341/04, Rec. 2011, pg. I-03813, apartado 54). Igualmente, el juez competente podrá adoptar las medidas provisionales o cautelares sobre bienes situados en otros Estados miembros; del mismo modo, el síndico provisional, nombrado con anterioridad a la apertura del procedimiento, también podrá actuar en los Estados miembros donde el deudor tenga establecimientos, adoptando medidas de seguridad previstas en la ley de dicho Estado (Considerando 16 Reglamento 1346).

23 J.L. Vallens, Petites affiches, $\mathrm{n}^{\circ}$ 18, 13 juin 2002, p. 20. En relación con estas últimas reglas de control de la decisión, el autor cita las siguientes: 1) la habilitación del síndico (arts. 19 y 53 del Reglamento de insolvencia 1346/2000); 2) la competencia del juez extranjero del lugar del centro de intereses principales del deudor a los efectos del exequátur de la decisión de apertura del procedimiento (art. 17 del Reglamento de insolvencia 1346/2000); 3) la cualidad de la jurisdicción competente en el sentido del artículo 2.d) del Reglamento 1346/2000; 4) el carácter ejecutorio de la decisión, y la posibilidad de suspender el procedimiento de ejecución de la resolución en caso de que en el Estado de origen se recurra la misma, tal como prescribe el artículo 37.1 del Reglamento 44/2001; 5) que la decisión no atente contra el orden público internacional del Estado requerido (art. 26 del Reglamento de insolvencia 1346/2000); 6) las características del deudor para que pueda ser sujeto de estos procedimientos de insolvencia del Reglamento; 7) la presencia de bienes del deudor sobre el territorio nacional; 8) la ausencia de un establecimiento del deudor en ese Estado que justifique la apertura de un procedimiento secundario en ese país (art. 17); 8) la ausencia de un procedimiento secundario en el Estado requerido (J.L. ValLENs, Petites affiches, $\mathrm{n}^{\mathrm{o}} 18,13$ juin 2002 , p. 21).

${ }^{24}$ El orden público al que hace referencia debe ser interpretado de la misma manera restrictiva que como se interpreta en el marco del anterior Convenio de Bruselas de 1968, actual Reglamento 1215/2012 (STJUE de 2 mayo 2006, Eurofood, C-341/04, Rec. 2006, pg. I-03813, apartado 62). De esta manera, debería alegarse la excepción de orden público para no reconocer una resolución en esta materia cuando dicho reconocimiento menoscabe un principio fundamental del ordenamiento del Estado requerido, esto es, cuando viole una norma jurídica que se considere fundamental en dicho país o cuando lesione un derecho, también fundamental, en dicho Estado (STJUE de 2 mayo 2006, Eurofood, C-341/04, Rec. 2006, pg. I-03813, apartado 63). En el asunto Eurofood, el Tribunal de Justicia consideró que se puede alegar orden público cuando la resolución a reconocer se ha 
los Estados miembros, también, no debían reconocer ni ejecutar decisiones que tuvieran por efecto una limitación de la libertad personal o del secreto postal (art. 25.3 Reglamento 1346).

\section{Ley aplicable a la determinación de los bienes sujetos al procedimiento de insolvencia}

21. La ley aplicable al procedimiento de insolvencia, ya sea éste principal o secundario, es la lex fori concursus, esto es, como ya se ha mencionado anteriormente, la ley del país donde se ha iniciado el procedimiento.

22. En este sentido, el tribunal remitente también pregunta por el Derecho aplicable a la determinación de los bienes sujetos al procedimiento secundario. Teniendo en cuenta los Considerandos 6 y 23 del Reglamento 1346/2000 -Considerandos 6 y 66 del Reglamento 848-, se puede interpretar que el legislador europeo entiende que las cuestiones que tengan normas de conflicto específicamente previstas para ellas en el Reglamento mencionado, deberán quedar reguladas por el mismo, sin embargo, para aquellas otras cuestiones que no sean objeto de tratamiento en el texto, el juez competente tendrá que aplicar sus normas de conflicto de Derecho internacional privado para determinar la ley reguladora de las mismas.

Pues bien, la lex fori concursus será la que determine, entre otras cuestiones, los bienes que forman parte de la masa activa ${ }^{25}$. En este sentido, el artículo 27.1 del Reglamento 1346 -artículo 34 del Reglamento 848- establece que los bienes del deudor afectados en el procedimiento secundario son los que se encuentren en el territorio del Estado donde se ha iniciado este procedimiento. Por tanto, el problema está en determinar los criterios de localización de los bienes.

23. Siguiendo el artículo 2.g) del Reglamento, el "«Estado miembro en el que se encuentre un bien» $[\mathrm{es}]^{26}$ :

- para los bienes materiales, el Estado miembro en cuyo territorio se encuentre el bien,

- para los bienes y derechos cuya propiedad o titularidad deba inscribirse en un registro público: el Estado miembro bajo cuya autoridad se lleve dicho registro,

- para los créditos: el Estado miembro en cuyo territorio se encuentre el centro de los intereses principales de su deudor, tal como se determina en el apartado 1 del artículo 3";

24. Por todo ello, el Tribunal de Justicia concluye que corresponde al juez del procedimiento secundario determinar, siguiendo los criterios anteriores, qué bienes se encuentran en el territorio del Estado del foro. Esos bienes que se encuentren, en nuestro caso, en Francia, serán los que estén sujetos al procedimiento secundario iniciado en este país.

El momento a tener en cuenta para determinar el lugar de situación de los bienes es el de apertura del procedimiento de insolvencia ${ }^{27}$.

\section{Conclusiones}

25. El caso objeto de estudio se plantea en relación con un procedimiento secundario de insolvencia abierto en Francia, encontrándose el procedimiento principal en curso en Reino Unido. En este esce-

\footnotetext{
dictado vulnerando el derecho fundamental a ser oído en el procedimiento de la persona afectada por el mismo (STJUE de 2 mayo 2006, Eurofood, C-341/04, Rec. 2006, pg. I-03813, apartado 67).

${ }_{25}$ M. Virgós Soriano/F. Garcimartín AlfÉrez, Comentario al Reglamento europeo de insolvencia", Thomson-Civitas, 2003, pp. 79-80; M. LLORENTE SÁnChEZ-ARJona, Tratamiento procesal de la insolvencia transfronteriza en la Unión Europea, Tirant Lo Blanch, Valencia, 2013, p. 103; M.J. Verdú CAÑEte, Procedimientos concursales comunitarios, Tirant Lo Blanch, Valencia, 2004, p. 79.

26 M. Virgós Soriano/F. Garcimartín Alférez, Comentario al Reglamento europeo de insolvencia”, Thomson-Civitas, 2003, pp. 164-167.

27 M. Virgós Soriano/F. Garcimartín Alférez, Comentario al Reglamento europeo de insolvencia”, Thomson-Civitas, 2003, p. 163; M. LloRente SÁnchez-ArJonA, Tratamiento procesal de la insolvencia transfronteriza en la Unión Europea, Tirant Lo Blanch, Valencia, 2013, p. 76.
} 
nario, el Tribunal de Justicia tiene que resolver dos cuestiones de Derecho Internacional Privado, cuales son, la competencia judicial internacional del juez del procedimiento secundario y el Derecho aplicable, en ambos casos, para determinar los bienes del deudor sujetos al procedimiento secundario de insolvencia.

26. En este sentido, comenzando por la primera, el Tribunal europeo, reiterando jurisprudencia anterior, afirma que el juez del procedimiento secundario es competente, no sólo para conocer de dicho procedimiento en sentido estricto, sino, también, para conocer de las cuestiones relacionadas con el mismo que pudieran plantearse. Entre estas cuestiones relacionadas se encuentra la determinación de los bienes del deudor que se encontrarían afectados en el procedimiento secundario y que, por tanto, escaparían del procedimiento principal.

27. Si bien es claro que la competencia para conocer de cuestiones anexas al procedimiento de insolvencia secundario también debe tenerla el juez que está conociendo de este procedimiento, es más controvertido que esa competencia tenga carácter alternativo o concurrente respecto de la que tiene el juez del procedimiento principal. En efecto, el Tribunal de Luxemburgo establece en la sentencia comentada que cuestiones relacionadas con el procedimiento secundario de competencia, tales como la determinación de los bienes del deudor que se deben tener en cuenta en este procedimiento, puedan ser determinadas tanto por el juez del procedimiento secundario como por el juez del procedimiento principal, de forma concurrente o alternativa, por tanto.

La razón que justifica esta afirmación para el Tribunal europeo es que esos bienes que se imputan al procedimiento secundario afectan al procedimiento principal porque suponen un detrimento de la masa activa en este último procedimiento. Si bien podemos estar de acuerdo con el argumento para justificar llegar a esta conclusión, también hay que tener en cuenta las consecuencias que puede conllevar la puesta en práctica de esta competencia concurrente. Esto es, es lógico que si dos tribunales distintos pueden pronunciarse sobre la misma cuestión, las resoluciones que dicten puedan ser contradictorias y, en el contexto internacional en el que nos movemos, puedan plantearse problemas de reconocimiento o ejecución de ambas en otro Estado.

En este sentido, consciente el legislador de esta posibilidad, se han regulado mecanismos de coordinación en el supuesto de que existan diversas resoluciones que resuelvan la misma cuestión. Así, el actual Reglamento de insolvencia recoge la tradicional regla de prior tempore potior iure, según la cual, la resolución dictada anteriormente será reconocida de pleno derecho en el resto de Estados miembros. Respecto de la ejecución, la norma remite al Reglamento 1215/2012 -texto que también recoge el principio prior tempore potior iure antes mencionado-.

28. Solucionar el problema de la competencia concurrente se plantea por la propia existencia de esta última, de tal modo que, si se estableciera la competencia exclusiva del juez del procedimiento secundario, no habría ninguna preocupación por la posibilidad de convivencia de resoluciones contradictorias en este tema. Por otro lado, el hecho de que el juez del procedimiento secundario tuviera la competencia exclusiva sobre las cuestiones conexas al mismo, no sería tan desproporcionado si partimos de la premisa de que tiene la competencia exclusiva en relación con el propio procedimiento secundario. Por lo tanto, sería razonable pensar que también pudiera tener la competencia exclusiva en relación con las cuestiones relacionadas con aquel.

29. Por último, en relación con el Derecho aplicable, el Tribunal de Justicia estipula que la ley reguladora de la determinación de los bienes del deudor es la lex fori concursus. Utilizando el principio de especialidad, el órgano judicial europeo afirma que la ley concursal será la que resuelva las cuestiones que ella misma recoge, dejando la regulación del resto de materias al Derecho que determinen las normas de conflicto del foro aplicables. Como el Reglamento de insolvencia regula la cuestión planteada de determinación de los bienes del deudor en el artículo 2.g), el juez del procedimiento secundario tendrá que seguir los criterios recogidos en este precepto para concretar los bienes del deudor que formarán parte de la masa activa en relación con este procedimiento secundario. 